\title{
Robot-resident neural networks: A step beyond computer simulation
}

\author{
FREDERICK J. BREMNER, WILLIAM COLLINS, and SARAH E. SCOTT \\ Trinity University, San Antonio, Texas
}

\begin{abstract}
A simple, commercially available robot platform is presented in this paper with suggested modifications for adding a neural network controller and a better sensory system. The robot, called HexWalker, is a six-legged, servo-driven, battery-powered platform. We found this platform easy to modify with both hardware and software changes. The software changes can be programmed from a standard PC and downloaded into the EEROMS of the robot. This robot provides a tangible model of sensory/motor integration which is useful in both teaching and research.
\end{abstract}

The long-range goal of the research described in this article is to build insect-like robots that are behaviorally and neuromorphically correct (Pearson \& Franklin, 1984) for use in both teaching and research. To proceed quickly on this project, we modified a commercially available robot (HexWalker) as our apparatus. Hex-Walker immediately provided us with ideas and limitations for the modification and design of our "on-board" neural controller. Within HexWalker's physical limits, we were able to implement a more neuromorphic network which successfully controlled its movements and integrated sensory input. HexWalker and its next generation example HexRay became a focal point of student interest. We capitalized on this interest to teach concepts such as degrees of freedom, matrix manipulation, sensory/motor integration, and controller design.

The omnibus controller that we use in our current robot is a hybrid of a motor component programmed as a production system and a sensory discrimination system designed around a neural network. In a sense this is a hardware version of the simulation published by Bremner and Gotts (1995), who used Braitenberg's Vehicle 5 (1984) as their inspiration. Vehicle 5 had two reversible motors and a visual transducer, but it was behaviorally limited by its serial memory. The computer simulation by Bremner and Gotts added distributed memory, which was resident in a neural network. The robot described in this study had six legs, three motors, and a sensory system composed of a tactile component and a sonar component. These components were all controlled by a Hopfield neural network. which in turn controlled a production system for motor control.

Many journal articles support the neuromorphology of robot arthropods (Beer, 1995; Beer, Ritzmann, \& Chiel, 1995; Pearson, 1976). Six-legged robots are very stable, very strong, and very adaptable. An insect often walks with

W. C. is in the Department of Engineering at Trinity. Correspondence concerning this paper should be addressed to F. J. Bremner, Department of Psychology, Trinity University, San Antonio, TX 78712 (e-mail: fbremner(atrinity.edu). a tripod gait, using the front and back legs on one side and the middle leg on the contralateral side. This was our robot's gait as well. Although insects have several tactile transducers, we chose antennae for tactile transduction. Whereas sonar-based sensory systems are more common in mammals (bats and whales), we chose sonar because the hardware was readily available. In short, we modified a commercially available six-legged robot with a distance transducer and a hybrid controller.

\section{METHOD}

\section{Apparatus}

HexWalker, a six-legged robot with a microcomputer and two sensory antennae, is purchasable from M \& T Systems, Huntington Beach, CA, as an unassembled kit (\$150). All components except for four AA, 6-V batteries are included. Motor movement (walking, turning, and backing) is powered by three servo motors developed by the hobby industry for use in radio-controlled airplanes and cars. One servo is connected to the middle legs, another to the front and back left legs, and the third to the front and back right legs. The artificial gait is achieved by two basic motions. The front and hind legs push forward or backward, while the middle legs shift the weight of the robot to the left or right. Thus the robot mass is always distributed between the front and hind legs on one side and the middle leg on the opposite side, simulating the typical tripod gait of insects.

The robot as purchased is controlled by a single miniature computer called the Basic StampI (Parallax Co.). It is preprogrammed to walk forward until striking an object with one of its antennae. Its reaction to a sensed object is a backing maneuver, followed by a turn. The resident program within the Basic Stamp can be modified or changed entirely with the use of a DOS-based PC and a software development kit (Digi-Key or Mondo-tronics).

The robot reported in this paper was modified by the authors to behave similarly to Braitenbergs's (1984) Vehicle 5 . This was done by adding (1) an ultrasound sensor (sonar) to give it distance perception and (2) an addi- 
tional microprocessor (Basic StamplI, Digi-Key, or Parallax) to control decision making. We used the Polaroid range finder (TI01) as the sonar transducer/receiver. The Basic StampII provided extended computational ability to support sensor processing and a Hopfield style neural network to control decision making. All motor control was programmed on Basic StampI, as was communication with Basic StampII. Our version of the robot, which we call Hexwalker/Radar or HexRay, has a new electronics (carrier) board that houses these modifications. This hardware can easily carry out real actions of hybrid simulation, such as those presented by Bremner and Gotts (1995).

\section{Procedure}

Our first step was to assemble the hardware of the robot platform. In particular, it takes some dexterity to put the legs, body, and servo motors together. HexWalker was first built according to the instructions provided with the kit. This gave us a walking robot that would back up and turn when an antenna was tripped. The robot has since been modified to fit the needs of the project. Sturdy aluminum legs have replaced the original plastic legs, which were prone to break. The supplied computer board was replaced with a larger board, which contained two CPUs (Basic StampI and II) and an edge connector for additional circuits.

The software development of the robot was divided into two tasks. A neural controller was needed for decision making, and low-level BASIC programming was required for the Basic Stamps. The neural controller was developed by training and testing various networks with standard applications packages such as BrainMaker (1990) and MicroSaint (1987). The neural model was then translated into BASIC code and downloaded into Basic Stampll. Basic Stampl was programmed to control the walking sequence. An additional sensing element was required for Vehicle 5 type behavior. A sensor range finder, not unlike radar, was added to the system. We used a T101 board, which was first developed by Polaroid and manufactured by Texas Instruments.

The send and receive commands for the sensor board required additional logic circuits so that the elapsed time of the reflected sound wave could be properly interfaced with the Basic Stamp functions. Because the Basic Stamps used are inexpensive ( $\$ 34$ to $\$ 50$ ) and can be plugged directly into the robot, we programmed several Basic Stamps with different task responsibilities. However it became apparent from the task analysis and the flow charts (Figure 1) that one chip for sensory processing and one chip for motor processing were most efficient.

\section{RESULTS}

The flow chart for the two Basic Stamps used to control HexRay is given in Figure 1. This controller emulated the behavior of Braitenberg's Vehicle 5 (1984). We first developed the controller as a standard three-layer backpropagation neural network. We used sensory neu-

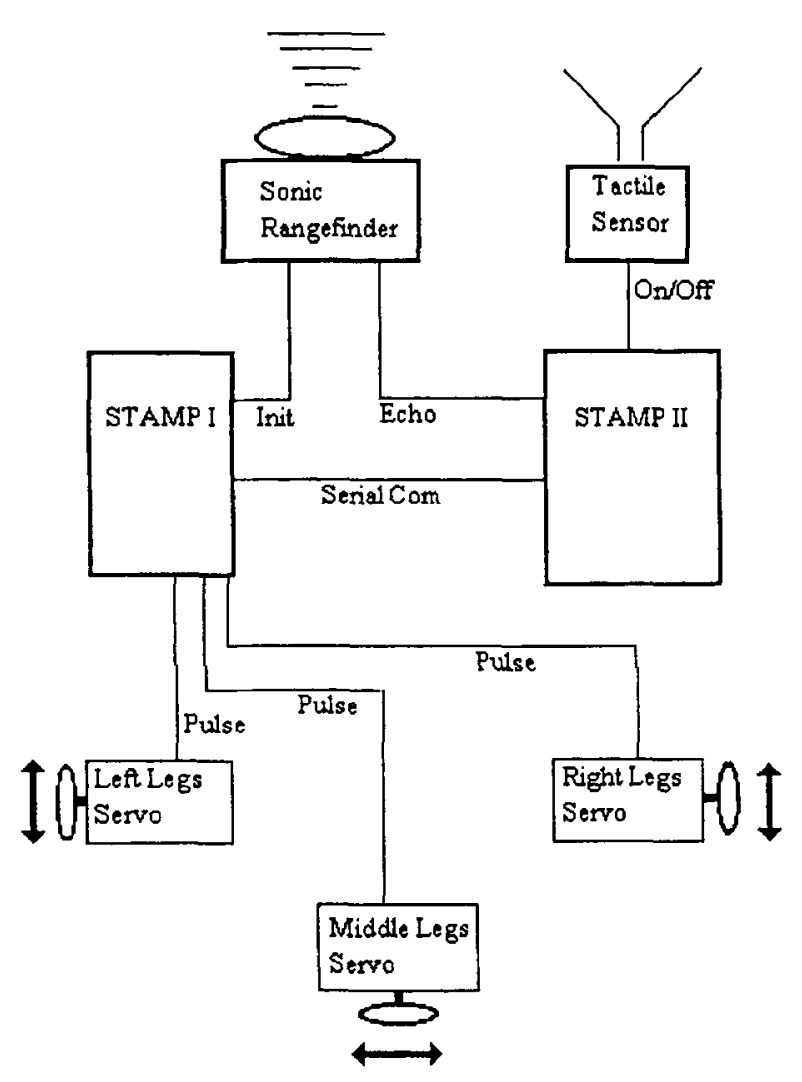

\section{HexRay Hardware Interface Diagram}

Figure 1. The flow chart for the two Basic Stamps used to control Hex Ray and the two input transducers.

rons as input and motor commands (forward, back, turn right, etc.) as output neurons. We found that a six-input, four-output network with 10 hidden neurons was sufficient to make correct decisions, but that it was too computationally intensive to be programmed into a Basic Stamp. This is primarily due to the overhead of floating point calculations required for such a design.

Instead of upgrading our computer hardware, we chose to explore other networks. We found that a Hopfield network using five or six neurons could be managed easily by the hardware of the Basic Stamp circuitry. Each neuron has only two states $(+1,-1)$. In this configuration, three neurons are related to sensory functions (object sighted, object near or far, antenna touching). The other neurons are associated with motor commands (forward, back, turn). A Hopfield network can be trained so that it relies only on a weight matrix of integers. Thus, computations "on board" the robot are integer multiplications and additions. The Hopfield schema that we employed takes as an initial vector the states of the sensory neurons along with zeros for the motor neurons. This vector is multiplied by the weight matrix (see Figure 3) until convergence is met. This is usually two repetitions in our case. The resulting value of the output neurons makes up the motor command. 
The two Basic Stamps interface via their $\mathrm{I} / \mathrm{O}$ pins configured as a serial communication port. This communication is not truly parallel but represents a strong handshaking between the two Basic Stamps.

Figure 2 is a flow chart of each Basic Stamp, showing the tasks that it performs and the order in which they are done. Basic StampII (right side of Figure 2), after an initialization period, is programmed to enter a repetitive loop which: (1) receives a ready command from Basic StampI (motor control), (2) checks sensor conditions (range and tactile), (3) calculates a Hopfield decision vector, (4) sends a command to motor control. Basic StampI (left side of Figure 2) does the following: (1) receives a motor command, (2) sends appropriate electrical signals to servos, (3) sends ready signal to Basic StampII (sensory processor), (4) sends initialization signal to range finder.

An expanded view of the functioning of the Hopfield neural network is given in Figure 3. We have developed networks with five or six neurons. Three neurons are related to sensory functions (object sighted, object distance, antenna sense). The other two or three neurons are associated with motor movements (walk forward, walk backward, turn). An input vector converged within two or three iterations whether there were five or six neurons in the neural network. Therefore processing time was not a limiting factor in HexRay.

The weight matrix for a five-neuron Hopfield network is shown in the lower portion of Figure 3. The input vector $(1-1100)$ from the sensory transducers is multiplied by the weight matrix to produce the output vector (1-1 $1-11$ ), which is sent to the motor control program.

\section{DISCUSSION}

One of the strengths of this research approach is that it can be used as a pedagogical tool. Thus our work shows the application of matrix algebra used by the Hopfield network. Even the shortcomings of HexRay have a use in teaching. For example, HexRay has only one degree of freedom on each leg, whereas it has been nicely demonstrated that each of the six legs of real insects has at least two degrees of freedom (Chiel \& Beer, 1989; Pearson, 1976). We use this discrepancy to initiate a discussion with our students on degrees of freedom as underlying various statistics, thus providing a "concrete" example of a difficult concept. On the other hand, the "tendons" that control HexWalker's legs give it a wiggle-waggle gait. In addition, insects have gaits other than the tripod gait, but

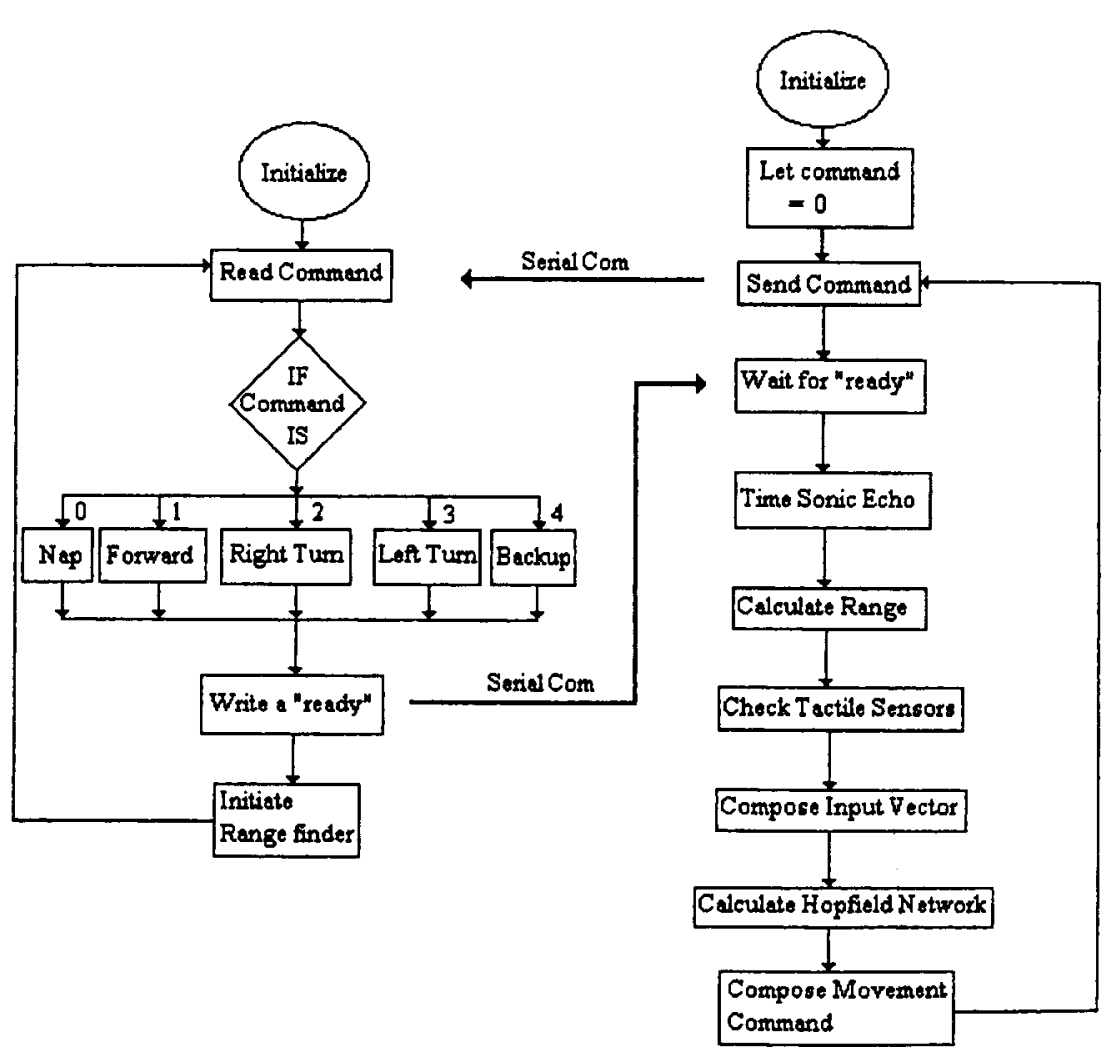

HexRay Sortware Flow Diagram

Figure 2. Detailed flow chart for Basic StampI (motor controller) and Basic StampII (sensory processor), showing the division of labor and the tasks that each performs. 


\section{Hopfield Network for HexRay}

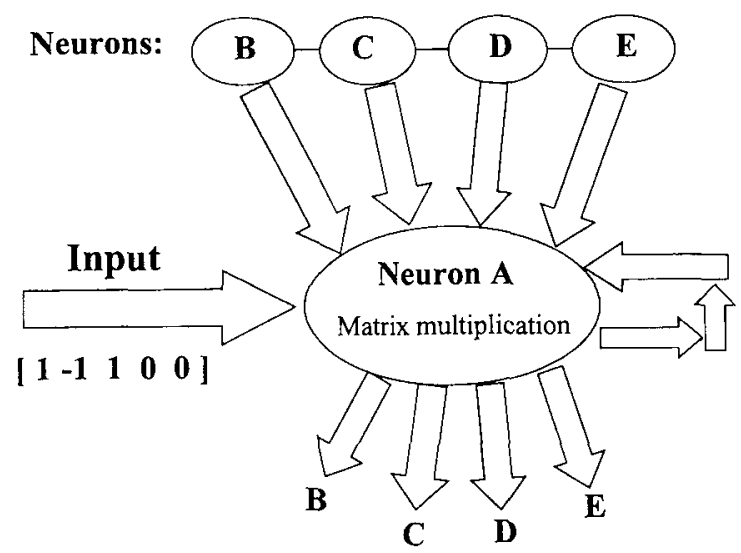

\begin{tabular}{|c|c|c|c|c|c|}
\hline Weight Matrix: & [ 5 & -1 & 1 & 1 & 3 \\
\hline & -1 & 5 & -1 & 3 & -3 \\
\hline & 1 & -1 & 5 & -3 & 3 \\
\hline & 1 & 3 & -3 & 5 & -1 \\
\hline & 3 & -3 & 3 & -1 & 5 \\
\hline
\end{tabular}

Output: [ $\left.\begin{array}{ccccc}1 & -1 & 1 & -1 & 1\end{array}\right]$

Figure 3. A diagram of the Hopfield network, showing the complete feedback of every neuron to neuron $A$ and of neuron $A$ to every neuron including itself. Note the input vector. The bottom portion of the figure contains the weight matrix of the five neurons and the resultant of multiplying the input vector by the weight matrix to yield the signum output vector.

it will take a more sophisticated robot platform to move more realistically.

In the future, we plan to test the robustness of our motor neural networks by lesioning them. The real nervous system controlling insect locomotion is a very flexible neural network which can recover from damage very quickly (Gallistel, 1980). One of the investigative techniques used to research the motor system is to degrade (brain damage) the nervous system of the robot to see whether these constructed motor networks show the same performance deficits as does the natural insect (Ya- mauchi, 1993). In addition, it has been demonstrated (Beer \& Gallagher, 1992) that in order to negotiate rough terrain there must be tactile sensory feedback to each leg. The next step in our project is to use the Prometheus robot platform as host to motor neural networks. Prometheus has 12 servos and two degrees of freedom on each leg. The hardware and software that we developed for HexRay are directly transportable to Prometheus. We intend to control each of the six legs with its own Basic Stamp, which in turn will be linked to a CPU for decision making. This arrangement will be a parallel distributed controller, much more neuromorphic than HexRay.

\section{REFERENCES}

BEER, R. D. (1995). A dynamical systems perspective on agentenvironment interaction. Artificial Intelligence, 72, 173- 215.

BeER, R. D., \& Gallagher, J. C. (1992). Evolving dynamical neural networks for adaptive behavior. Adaptive Behavior, 1, 91-121.

Beer, R. D., RitzmanN, R. E., \& Chiel, H. J. (1995). Models of the neural basis of the insect behavior. In S. Zornetzer, J. Davis, C. Lau, \& T. McKenna (Eds.), An introduction to neural and electronic networks (2nd ed.). New York: Academic Press.

Brainmaker Professional Version 2.0 [Computer program] (1990). Grass Valley, CA: California Scientific Software.

BRAITENBERG, V. (1984). Vehicles: Experiments in synthetic psychology. Cambridge, MA: MIT Press.

BremNer, F. J., \& GotTS S. J. (1995). Shades of Braitenberg's Vehicle 5. Behavior Research Methods, Instruments, and Computers, 27, 169172.

ChIEl, J. C., \& BeER, R. D. (1989). Lesion study of a heterogeneous artificial neural network for hexapod locomotion. Proceedings of the International Journal Conference Neural Networks, 1, 407-414

GaLLISTEL, C. R. (1980). The organization of action: A new synthesis. Hillsdale, NJ: Erlbaum.

MicroSaint [Computer program] (1987). Boulder, CO: Micro Analysis \& Design, Inc.

Pearson, K. [G.] (1976, December). The control of walking. Scientific American, 235(6) 72-82.

Pearson, K. G., \& Franklin, R. (1984). Characteristics of leg movements and patterns of coordination in locusts walking on rough terrain. International Journal of Robotics Research, 3, 101-112.

YAMAUCHI, B. M. (1993). Dvnamical neural networks for mobile robot control (NRL Memorandum Report AIC-033-93). Washington, DC Naval Research Laboratory.

(Manuscript received November 4, 1996: revision accepted for publication February 5, 1997.) 